\title{
APLICAÇÃO DE $\alpha$-OXOCETENO DITIOCETAIS EM SÍNTESE ORGÂNICA
}

Geonir M. Siqueira, Patrícia D. Neuenfeldt, Anaí Duarte, Lizandra C. Bretanha, Rogério A. Freitag e Wilson Cunico* Departamento de Química Orgânica, Instituto de Química e Geociências, Universidade Federal de Pelotas, CP 354, 96010-900 Pelotas - RS, Brasil

Recebido em 16/7/09; aceito em 1/10/09; publicado na web em 10/3/10

REACTIVITY OF $\alpha$-OXOKETENE DITHIOCETALS IN ORGANIC SYNTHESIS. The synthesis and reactivity of $\alpha$-oxoketenes dithioacetals ( $S, S$-acetals), general structure $\left[\left(\mathrm{R}^{1} \mathrm{C}=\mathrm{OC}\left(\mathrm{R}^{2}\right)=\mathrm{C}\left(\mathrm{SR}^{3}\right)\left(\mathrm{SR}^{4}\right)\right]\right.$, are reported. We also showed the application of $S, S$ acetals as synthons for efficient synthesis of isoxazoles, pyrazoles, indazoles, thiophenes, dithiol thiones, pyridines, pyrimidines and other heterocycles. This work aims to review the importance of $\alpha$-oxoketenes dithioacetals in organic chemistry during the past few years.

Keywords: $S, S$-acetals; heterocycles; $\alpha$-oxoketene dithioacetals.

\section{INTRODUÇÃO}

A síntese de compostos contendo átomos de enxofre é de grande interesse em química orgânica, tanto pelo seu uso como intermediário sintético ou pelo seu potencial biológico. Como exemplo de aplicações farmacológicas, vários compostos têm sido usados como agentes antibacterianos, ${ }^{1}$ agentes anti- HIV, ${ }^{2}$ agentes no combate ao câncer de fígado ${ }^{3}$ entre outras doenças.

Este trabalho teve como objetivo mostrar a importância de métodos de síntese e de modificação de cadeia de $\alpha$-oxoceteno ditioacetais bem como explicitar sua aplicação como precursor de heterociclos com potencial ação biológica. Assim, as metodologias apresentadas priorizam técnicas que permitem a introdução direta de átomos de enxofre na estrutura de heterociclos, bem como sua presença como grupo substituinte (tióis). Serão mostradas técnicas de obtenção de $\alpha$-oxoceteno ditioacetais, a partir da reação de cetonas com hidretos metálicos $(\mathrm{NaH}, \mathrm{KH})$ e outros tipos de bases na presença de $\mathrm{CS}_{2}$ com posterior adição de haleto de alquila apropriado. Esta revisão também visa mostrar a reatividade de $\alpha$-oxoceteno ditioacetais e sua utilização como precursor na síntese de heterociclos: piridinas, pirazóis, isoxazóis, tiofenos, benzotiofenos, indazóis, pirimidinas, naftalenos, ditioltionas, pirazolopiridinas e piridoindóis.

\section{COMPOSTOS $\alpha$-OXOCETENO DITIOACETAIS}

Os $\alpha$-oxoceteno ditioacetais são cetonas $\alpha, \beta$-insaturadas por grupos sulfurados na posição $\beta$, sendo compostos de alta importância biológica e versatilidade sintética. Observa-se uma procura de novas metodologias para a síntese e aplicação, visando o aprimoramento de técnicas e formação de novos compostos. A Figura 1 representa a estrutura geral dos $\alpha$-oxoceteno ditioacetais, com substituintes $\mathrm{R}^{1}, \mathrm{R}^{2}, \mathrm{R}^{3}$ e $\mathrm{R}^{4}$ sendo grupos alquila, arila ou hidrogênio.

$$
\overbrace{\mathrm{R}^{2}}^{\mathrm{SR} \mathrm{R}^{3}}
$$

Figura 1. Estrutura geral de $\alpha$-oxoceteno ditioacetais

\footnotetext{
*e-mail: wilson.cunico@ufpel.edu.br
}

\section{Síntese de $\alpha$-oxoceteno ditioacetal}

$\mathrm{Na}$ literatura são encontradas basicamente duas metodologias para a síntese dos $\alpha$-oxoceteno ditioacetais. A primeira baseia-se na reação envolvendo um agente acilante, uma base (geralmente piridina) e o tritio-ortoacetato (rota A) (Esquema 1). A segunda metodologia baseia-se na reação do enolato proveniente de um composto carbonílico que possui hidrogênio $\alpha$-carbonila com o dissulfeto de carbono $\left(\mathrm{CS}_{2}\right)($ rota B) (Esquema 1)

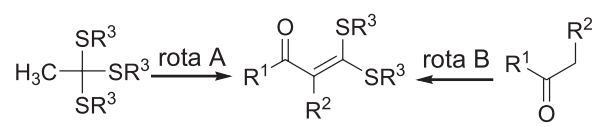

Esquema 1.

Sintese de $\alpha$-oxoceteno ditioacetais a partir do tritio-ortoacetatos

Hojo e Masuda, ${ }^{4}$ em 1975, realizaram a síntese dos tri-haloooxoceteno ditioacetais 3 com reações envolvendo o uso de anidrido tri-haloacético $(\mathrm{X}=\mathrm{F}, \mathrm{Cl})$, piridina e tio-ortoacetato de triarila $\mathbf{1 e m}$ uma proporção $2: 2: 1$, éter ou clorofórmio e tempo reacional de 24 h (Esquema 2). Segundo os autores, a formação do intermediário 2 possibilita formação dos produtos, através do ataque nucleofílico ao reagente acilante.

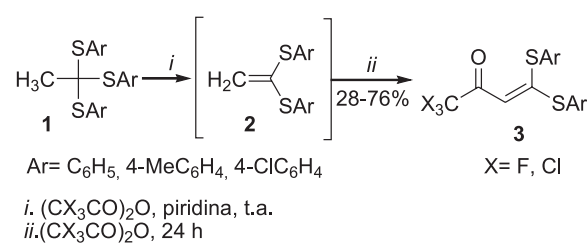

Esquema 2.

Síntese de $\alpha$-oxoceteno ditioacetais a partir de compostos carbonílicos

Ali e Tanimoto ${ }^{5}$ sintetizaram $\alpha$-oxoceteno ditioacetais partindo de ésteres 4. Os precursores foram tratados com litiodi-isopropilamina (LDA) com formação do enolato in situ, o dissulfeto de carbono $\left(\mathrm{CS}_{2}\right)$ foi adicionado e com a posterior adição de iodeto (brometo) de alquila (Esquema 3). Os compostos 5 foram obtidos em bons rendimentos.

Mahata e colaboradores, ${ }^{6}$ em 2003, sintetizaram o 4,4-dimetil-1,1dimetoxi-3-buten-2-ona 7 a partir da reação do enolato do piruvaldeído 


$$
\begin{aligned}
& \underset{79-87 \%}{\rightarrow} \mathrm{R}_{\mathrm{R}^{3} \mathrm{O}} \\
& \mathrm{R}_{\mathbf{S}}
\end{aligned}
$$

Esquema 3.

dimetilacetal 6 com o $\mathrm{CS}_{2}$ e iodeto de metila, obtendo o produto com $85 \%$ de rendimento (Esquema 4 ). A reação ocorre através do ataque do enolato (formado pela perda do próton da metila $\alpha$ à carbonila do piruvaldeído dimetilacetal) ao carbono parcialmente positivo do $\mathrm{CS}_{2}$, seguida de alquilação dos dois átomos de enxofre pelo iodeto de metila. O trabalho apresenta o potencial sintético do composto 7 como precursor para a síntese de heterociclos de 5 e 6 membros.

$$
\begin{aligned}
& (\mathrm{MeO})_{2} \mathrm{HC}_{6} \\
& { }_{6}^{\mathrm{O}} \\
& \text { i. } \mathrm{NaH} / \mathrm{THF} \\
& \text { ii. } \mathrm{CS}_{2} \\
& \text { iii.Mel (2eqv) }
\end{aligned}
$$

Esquema 4.

Recentemente, Abdelhaim e colaboradores ${ }^{7}$ sintetizaram $\alpha$-oxoceteno ditioacetais 9 tendo como precursor o esteroide epiandrosterona $\mathbf{8}$ utilizando diferentes agentes alquilantes e acilantes em bons rendimentos (Esquema 5). Utilizaram os compostos do tipo 9 como intermediários na obtenção dos heterociclos tiofenos, benzoxazóis, benzoimidazóis e pirimidinas, potentes agentes antibacterianos.

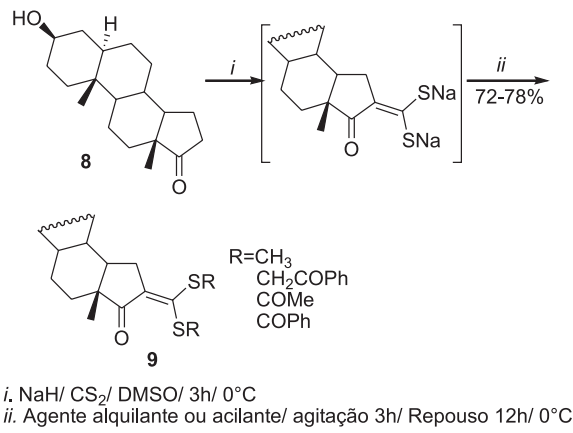

Esquema 5.

\section{Reatividade de $\alpha$-oxoceteno ditioacetais}

Os $\alpha$-oxoceteno ditioacetais são compostos de alta versatilidade sintética. Possuem dois centros eletrofilicos, o carbono da carbonila e o carbono $\beta$ à carbonila, e dois centros nucleofílicos, o carbono $\alpha$ à carbonila e o oxigênio da carbonila (Figura 2). Além disso, possuem o grupo SR que pode atuar como grupo abandonador em reações de adição-eliminação no carbono $\beta$. A reatividade de $\alpha$-oxoceteno ditioacetais $(S, S$-acetais) é semelhante à reatividade de $\beta$-dialcoxivinilcetonas $(\mathrm{O}, \mathrm{O} \text {-acetais })^{8}$ e de enaminonas $(\mathrm{N}, \mathrm{O}$-acetais $){ }^{9}$

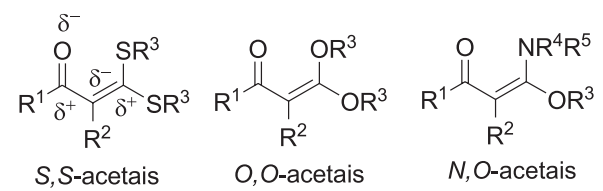

Figura 2. Centros reativos de $\alpha$-oxoceteno ditioacetais (S,S-acetais)
A Figura 3 mostra a variedade de reações que podem ocorrer em $\alpha$-oxoceteno ditioacetais. Assim, os $\alpha$-oxocetenos podem sofrer reações de adição à carbonila, adição-eliminação na carbonila, adição ao carbono $\beta$ à carbonila, adição-eliminação no carbono $\beta$, substituição eletrofílica no carbono $\alpha$, redução da carbonila e redução da dupla ligação.
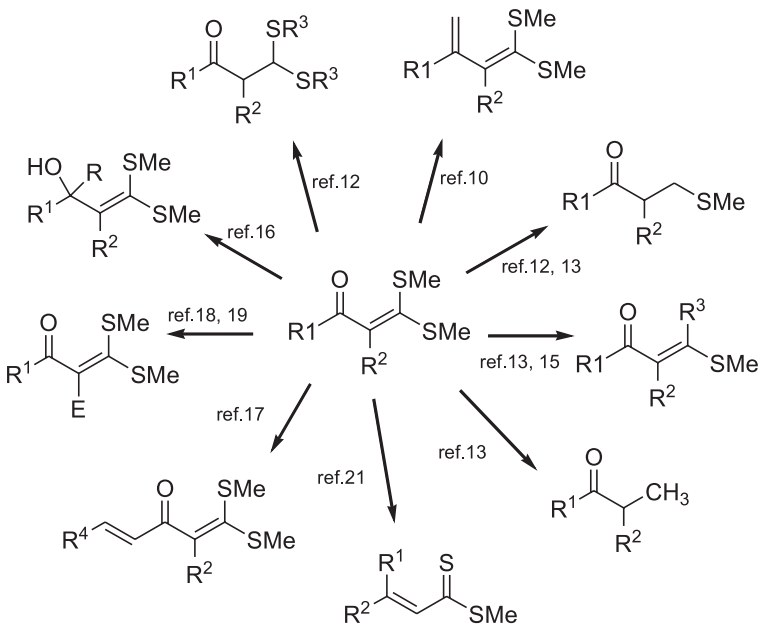

Figura 3. Modificações químicas em $\alpha$-oxoceteno ditioacetais

\section{Redução de $\alpha$-oxoceteno ditioacetais}

$\alpha$-Oxoceteno ditioacetais $\mathbf{1 0}$ foram estudados frente a reações de olefinação de Witting para formar os compostos $\mathbf{1 1}^{10}$ (Esquema 6). Os dienos $\mathbf{1 1}$ foram utilizados como precursores em reações Diels-Alder. ${ }^{11}$

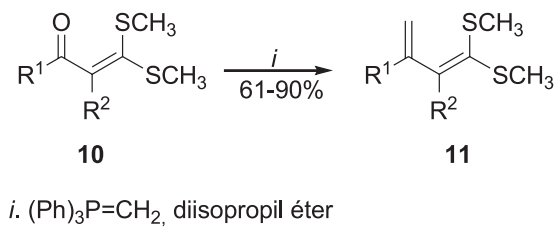

\section{Esquema 6.}

Gammill e colaboradores ${ }^{12}$ mostraram a redução seletiva de $\alpha$-oxoceteno ditioacetais $\mathbf{1 2}$ em contato com hidreto de di-isobutilalumínio (DIBAL), produzindo as cetonas ditioalquiladas $\mathbf{1 4}$ (redução seletiva da dupla ligação), e com 9-borabiciclo[3,3,1]nonano (9-BBN), formando as cetonas tioalquiladas $\mathbf{1 3}$ (redução da dupla ligação e de um grupo tiometil) (Esquema 7).

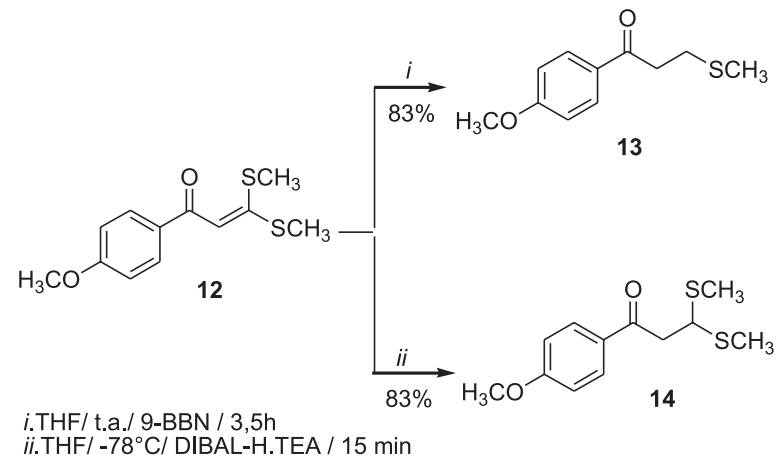

Esquema 7.

Yadav e colaboradores ${ }^{13}$ produziram $\beta$-metiltiometileno cetonas 16, $\beta$-tiometilcetonas 17 e $\alpha$-metilcetonas 18 através da redução de 
$\alpha$-oxoceteno ditioacetais 15 com $\mathrm{Zn}$ e $\mathrm{ZnCl}_{2}$-TMEDA $\left(N, N, N^{\prime}, N^{\prime}\right.$ tetrametiletilenodiamina). O produto obtido depende da quantidade do agente redutor utilizado, por exemplo, com 1,5 equivalentes apenas um grupo SMe é reduzido; com 3 equivalentes um grupo SMe e a dupla ligação são reduzidos; e com 5 equivalentes os dois grupos SMe e a dupla ligação são reduzidos (Esquema 8).

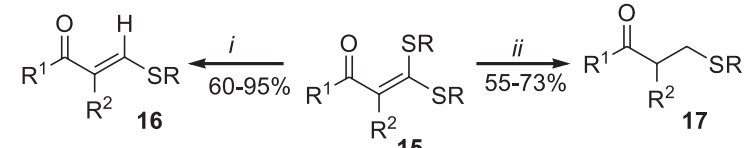

$$
\begin{aligned}
& \text { i. } \mathrm{Zn} / \mathrm{ZnCl}_{2} \text {-TMEDA (1,5 eqv) } 15 \text { ii. } \mathrm{Zn} / \mathrm{ZnCl}_{2} \text {-TMEDA (3 eqv) } \\
& \text { EtOH, Refluxo, 3-7h } \quad 50-\left.85 \%\right|_{i i i} \quad \text { EtOH, Refluxo, 7-12h } \\
& \begin{array}{ll}
\hline \mathrm{R}^{1} & \mathrm{R}^{2} \\
\hline \mathrm{C}_{6} \mathrm{H}_{5} & \mathrm{H} \\
\mathrm{C}_{6} \mathrm{H}_{5} & \mathrm{CH}_{3} \\
& \mathrm{H} \\
\mathrm{CH} & \mathrm{H} \\
\mathrm{CH}_{3} & \mathrm{CH}_{3} \\
\mathrm{CH}_{3} & \mathrm{H} \\
\multicolumn{2}{c}{\left(\mathrm{CH}_{2}\right)_{4^{-}}}
\end{array} \\
& \text {iii. } \mathrm{Zn} / \mathrm{ZnCl}_{2} \text {-TMEDA (5 eqv) } \\
& R^{1}{\stackrel{\mathrm{O}}{\mathrm{R}^{2}}}^{\mathrm{Me}} \mathrm{Me} \\
& \begin{array}{r}
R^{2} \\
18
\end{array}
\end{aligned}
$$

Esquema 8.

Reações de adição de compostos organo-magnésio, organo-lítio e organo-cobre em $\alpha$-oxocetenos ditioacetais

Reações com organo-cobre promovem o ataque seletivo ao carbono $\beta$ carbonila de $\alpha$-oxocetenos ditioacetais com a eliminação de um dos grupos tioalquil. ${ }^{14}$ Mehta e colaboradores ${ }^{15}$ testaram duas condições reacionais para a adição de reagente benzilcobre em $\alpha$-oxoceteno ditioacetais. Os $\alpha$-oxoceteno ditioacetais 19 sofreram adição no carbono $\beta$ com $\mathrm{R}^{2} \mathrm{CH}_{2} \mathrm{Cu}(\mathrm{X}) \mathrm{MgCl}$ dando produtos 20 com a configuração exclusiva $Z$ (Esquema 9); nenhum vestígio do estereoisômero $E$ ou da substituição do segundo grupo tiometil foi observado. Os autores constataram que na presença dos reagentes clorotrimetilsilano (TMSCl) e $N, N, N, N$-tetrametiletilenodiamina (TMEDA) houve um acréscimo no rendimento das reações.

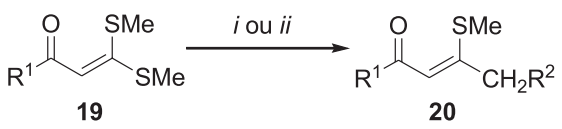

i. $\mathrm{R}^{2} \mathrm{CH}_{2} \mathrm{MgCl} / \mathrm{CuCl} / \mathrm{Et}_{2} \mathrm{O}-\mathrm{THF} /-78^{\circ} \mathrm{C}(57-68 \%)$ ii. $\mathrm{R}^{2} \mathrm{CH}_{2} \mathrm{MgCl} / \mathrm{CuCl} / \mathrm{TMSCl} / \mathrm{TMEDA}$ $/ \mathrm{Et}_{2} \mathrm{O}-\mathrm{THF} /-78^{\circ} \mathrm{C}(69-87 \%)$

Esquema 9.

Yadav e colaboradores ${ }^{16}$ estudaram as reações de adição de compostos organo-magnésio e organo-lítio em $\alpha$-oxocetenos ditioacetais 21 (Esquema 10). Mostraram que a reação dos organo-lítios preparados a partir do tolueno $(\mathrm{R}=\mathrm{H})$ e do $m$-xileno $\left(\mathrm{R}=\mathrm{CH}_{3}\right)$ frente aos compostos $\mathbf{2 1}$ produziram apenas os produtos de adição à carbonila (produto 23), do mesmo modo que a reação com o organo-magnésio (produto 22). Já a reação com o $o$-xileno formou produto de adição do organo-lítio à carbonila e ao carbono $\beta$ (produto 24 ).

\section{Reação de condensação aldólica em $\alpha$-oxocetenos ditioacetais}

Os $\alpha$-oxoceteno ditioacetais aromáticos $\mathbf{2 7}$ foram obtidos com o intuito de estudar sua ação contra a leishmania. ${ }^{17}$ Apesar de possuírem atividade antileishmaniose moderada, são intermediários versáteis na síntese de uma variedade de novas moléculas. Os compostos 27 foram obtidos a partir da reação de condensação aldólica Claisen-Schmidt do enolato formado in situ (reação do 4,4-ditiometil-3-buten-2-ona 25 com $\mathrm{KOH}$ ) com os benzaldeídos substituídos 26 (Esquema 11).

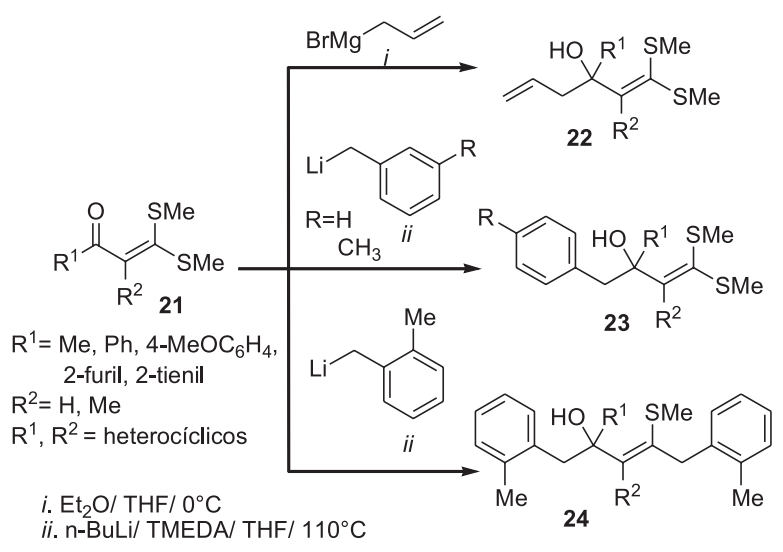

Esquema 10.

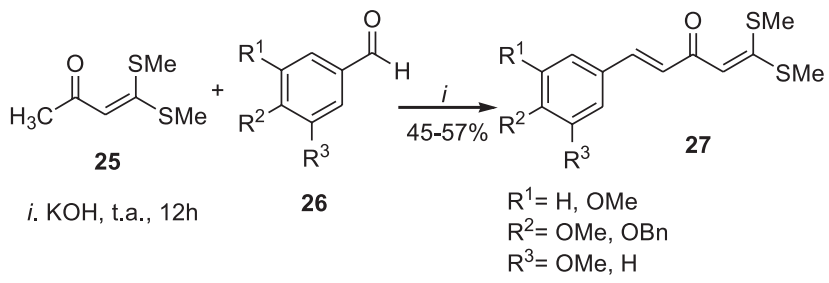

Esquema 11.

Reações de substituição eletrofílica no carbono $\alpha$ à carbonila

Yamamoto e colaboradores ${ }^{18}$ descreveram a adição de bromo ao carbono $\alpha$ carbonila de $\alpha$-oxoceteno ditioacetais 28 isolando os $\alpha$-bromo- $\beta$-oxoceteno ditioacetais 29 (Esquema 12). A reação dos compostos bromados com hidreto de tributilestanho e azobisisobutironitrila (AIBN) produz as lactonas $\mathbf{3 0}$ com rendimentos de moderados a bons.

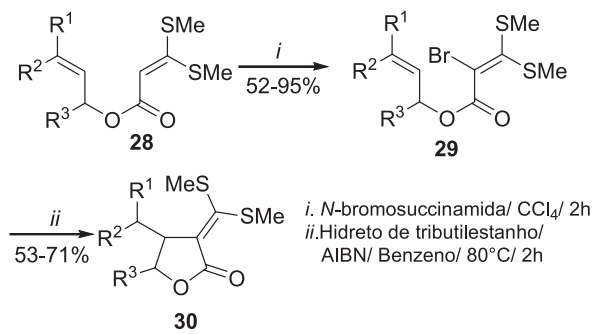

Esquema 12.

Anabha e Asokan ${ }^{19}$ estudaram a reação de formilação no carbono $\alpha$ carbonila de $\alpha$-oxoceteno ditioacetais. Tal procedimento foi realizado por meio da reação do arilceteno ditioacetal $\mathbf{3 1}$ com reagente de Vilsmeier-Haack (DMF-POCl ${ }_{3}$ ), formando o 2-aroil-3,3-ditiometil acrilaldeído 32 (Esquema 13).
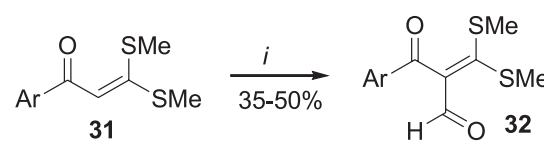

$\mathrm{Ar}=\mathrm{C}_{6} \mathrm{H}_{5}, 4-\mathrm{MeC}_{6} \mathrm{H}_{4} 4-\mathrm{BrC}_{6} \mathrm{H}_{4}$ 4- $\mathrm{MeOC}_{6} \mathrm{H}_{4}$ 3- $\mathrm{MeOC}_{6} \mathrm{H}_{4}, 2,3-(\mathrm{OMe})_{2} \mathrm{C}_{6} \mathrm{H}_{4}, 4-\mathrm{NO}_{2} \mathrm{C}_{6} \mathrm{H}_{4}$, 2-Naftil

i. a) $\mathrm{DMF} / \mathrm{POCl}_{3}$ b) $\mathrm{K}_{2} \mathrm{CO}_{3} / 100^{\circ} \mathrm{C} / 20 \mathrm{~h}$

Esquema 13.

\section{Adição-eliminação na carbonila}

Ortega-Jiménez ${ }^{20}$ e colaboradores sintetizaram complexos de paládio derivados do $\alpha$-difenil-hidrazinoxoceteno ditioacetais 34 
(Esquema 14). Os ligantes $\mathbf{3 4}$ foram obtidos através da reação entre os $\alpha$-xoceteno ditioacetais 33 e $N, N$-difenil-hidrazina. Os compostos 34 quando em contato com $\mathrm{Na}_{2}\left[\mathrm{PdCl}_{4}\right]$ formam os complexos ortopaládio 35 caracterizados por difração de raio-X. Os complexos 35 foram estudados em reações de trasmetalação.
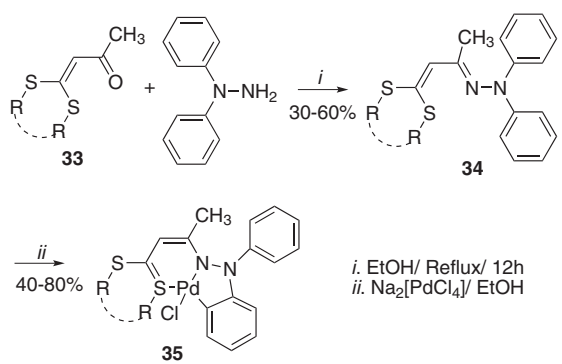

Esquema 14

\section{Formação de tiol ésteres}

Nair e colaboradores ${ }^{21}$ sintetizaram tiol ésteres $\alpha, \beta$-insaturados 37 a partir da reação entre os $\alpha$-oxoceteno ditioacetais 36 com o reagente de Lawesson, obtendo bons rendimentos (Esquema 15). Tiol ésteres também podem ser sintetizados tendo como precursor $\alpha$-hidroxiceteno ditioacetais e o reagente da Lawesson.

$$
i: \mathrm{C}_{6} \mathrm{H}_{6} \text {, refluxo, } 1 \mathrm{~h}
$$

Esquema 15.

\section{Síntese de compostos heterocíclicos a partir de $\alpha$-oxoceteno ditioacetais}

Os $\alpha$-oxocetenos ditioacetais são excelentes precursores na síntese de heterociclos com potencial atividade biológica. Alguns exemplos são apresentados na Figura 4. Vários heterociclos contendo o átomo de enxofre têm sido usados como agente antibacteriano, agente antiviral e no combate ao câncer como, por exemplo, o oltipraz 38, um representante da classe das 1,2-ditiol-3-tionas, que tem se mostrado eficiente no tratamento do câncer de fígado, ${ }^{22} \mathrm{o}$ tioconazol 40, composto contendo um tiofeno em sua estrutura, possuindo atividade antifúngica; ${ }^{23}$ a emtricitabina 42 (-FTC, Emtriva ${ }^{\circledR}$ ) que é um antirretroviral que atua na inibição da enzima transcriptase reversa do HIV, impedindo a replicação viral ${ }^{2}$ o racivir consiste na mistura 1:1 do agente anti-HIV -FTC (emtricitabina) 42 e de seu enantiômero +FTC 41, sendo mais ativo que os isômeros puros, inclusive apresentando ação contra o vírus da hepatite tipo B. O racivir atualmente

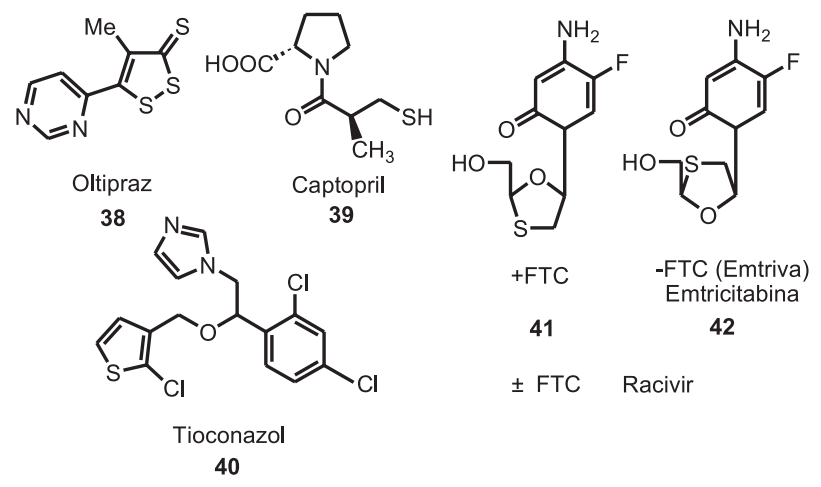

Figura 4. Heterociclos contendo enxofre com ação biológica comprovada encontra-se em fase clínica II contra o vírus HIV.

Além dos heterociclos contendo enxofre serem importantes para o estudo farmacológico, o grupo tiol também apresenta importância na química medicinal. Por exemplo, o captopril 39 que é um potente hipertensivo atualmente em uso clínico. ${ }^{24}$

Assim como as $\beta$-dialcoxivinil cetonas $(O, O$-acetais $),{ }^{25}$ e os $N, O$ acetais (enaminonas), ${ }^{26}$ os $\alpha$-oxoceteno ditioacetais ( $S, S$-acetais) são versáteis precursores para a síntese de diversos heterociclos de 5 e de 6 membros, conforme apresentados na Figura 5.

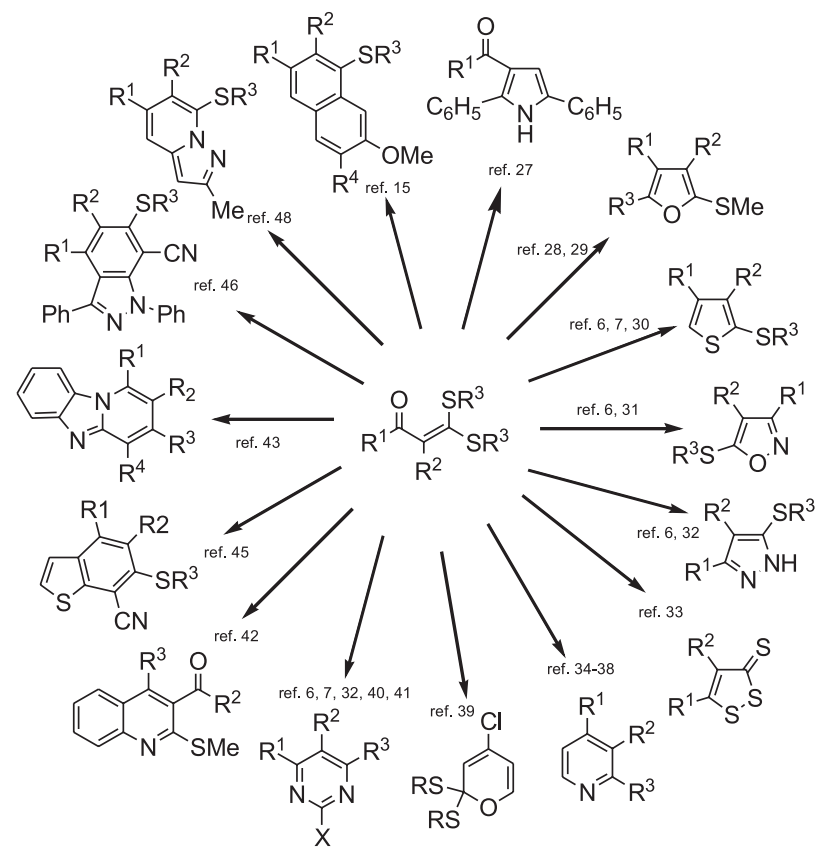

Figura 5. Compostos heterocíclicos derivados de $\alpha$-oxoceteno ditioacetais

\section{Síntese de anéis de cinco membros}

\section{Sintese de pirróis}

Balu e colaboradores ${ }^{27}$ sintetizaram pirróis $\mathbf{4 5}$ atráves da cicloadição [3+2] de $\alpha$-oxoceteno ditioacetais 43 com 1,3-difenil-2-azoalillítio 44 (Esquema 16). Os grupos tiometilas atuam como grupos abandonadores promovendo a aromatização do sistema.

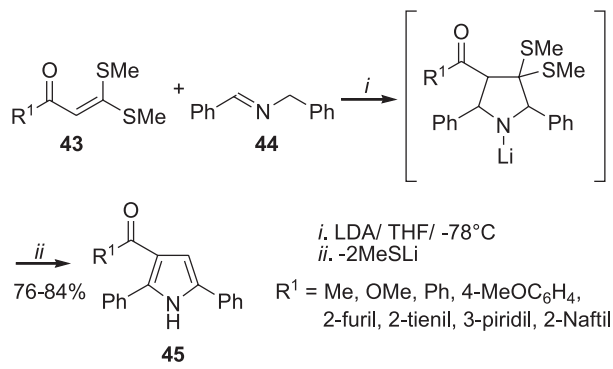

Esquema 16.

Síntese de furanos

Okazaki e colaboradores ${ }^{28}$ sintetizaram tiometilfuranos 48 pela reação de $\alpha$-oxoceteno ditioacetais $\mathbf{4 6}$ com o metileto de dimetilsulfônio passando pelos intermediários 2,2-bis(tiometil)-2,5-di-hidrofuranos 47 (Esquema 17). Os tiometilfuranos 47 podem ser obtidos através de três metodologias: solução de $\mathrm{HCl}$ catalítico (condição a); cromatografia em coluna florisil (condição b); ou reação com iodeto de metila (condição c). Tiometilfuranos provenientes de $\alpha$-oxoceteno ditioacetais também podem ser obtidos a partir da condensação de Darzen com o litiobromo acetato de etila em rendimentos moderados. ${ }^{29}$ 


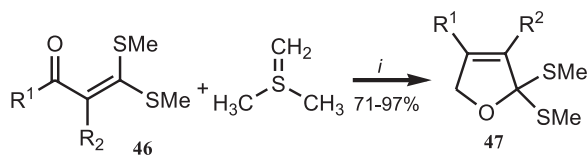

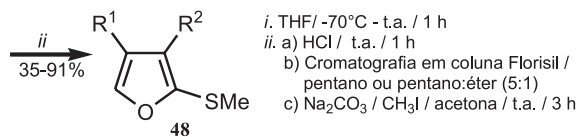

Esquema 17.

\section{Sintese de tiofenos}

Explorando o potencial sintético do composto 7, o 2-(tiometil) tiofen-4-il]carbaldeído 49 foi obtido a partir de uma suspensão $\mathrm{Zn}-\mathrm{Cu}$ em éter seco e cristais de iodo (Esquema 18$),{ }^{6}$ condições reacionais semelhante às utilizadas por Bhat e colaboradores ${ }^{30}$ na síntese de uma gama de tiofenos derivados de $\alpha$-oxoceteno ditioacetais.
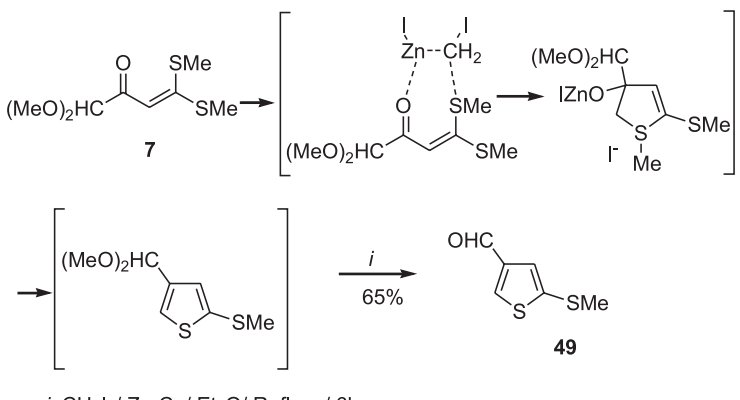

i. $\mathrm{CH}_{2} \mathrm{I}_{2} / \mathrm{Zn}-\mathrm{Cu} / \mathrm{Et}_{2} \mathrm{O} /$ Refluxo/ $6 \mathrm{~h}$

Esquema 18.

Abdelhaim e colaboradores ${ }^{7}$ sintetizaram tiofenos $\mathbf{5 0}$ a partir da ciclização intramolecular de $\alpha$-oxocetenos ditioacetais 9 derivados do esteroide epiandrosterona (Esquema 19).

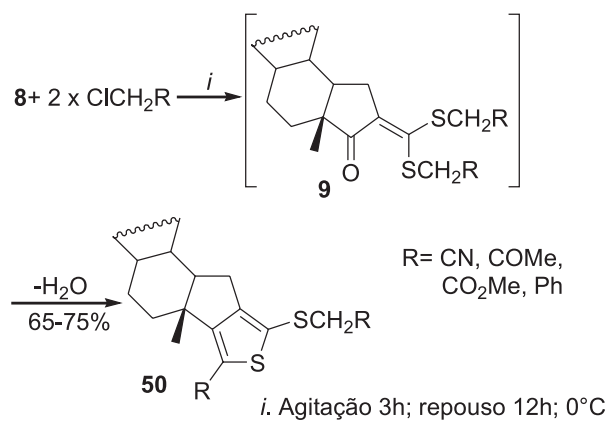

Esquema 19.

\section{Sintese de isoxazóis e pirazóis}

Banerjee e colaboradores ${ }^{31}$ sintetizaram tiometilisoxazóis $\mathbf{5 2}$ com atividade antiparasitária, através da reação de $\alpha$-oxoceteno ditioacetais 51 com cloridrato de hidroxilamina (Esquema 20). A reação procede com o ataque do nitrogênio da hidroxilamina no carbono $\beta$ e posterior ciclização intramolecular com o oxigênio da hidroxilamiana atacando a carbonila dos $\alpha$-oxoceteno ditiometilacetais, ocorrendo a ciclização e a eliminação de água. Os compostos apresentaram atividade contra $A$. ceylanicum e $N$. dubius in vitro e contra a $N$. dubius in vivo.

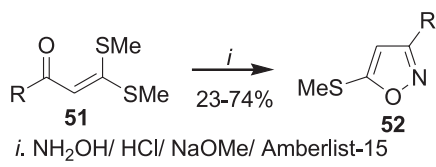

Esquema 20.
Mahata e colaboradores ${ }^{6}$ sintetizaram o 5-tiometilisoxazol 53 e o (3)5-tiometilpirazol $\mathbf{5 4}$ com o substituinte aldeído protegido na forma de acetal, a partir da reação entre o 4,4-ditiometil-1,1-dimetoxi-3buten-2-ona $7 \mathrm{em}$ etanol com cloridrato de hidroxilamina e hidrato de hidrazina, respectivamente (Esquema 21). A desproteção do acetal com ácido acético produz o [(3)5-tiometil-1H-pirazol-(5)3-il] carbaldeído 55 em ótimo rendimento.

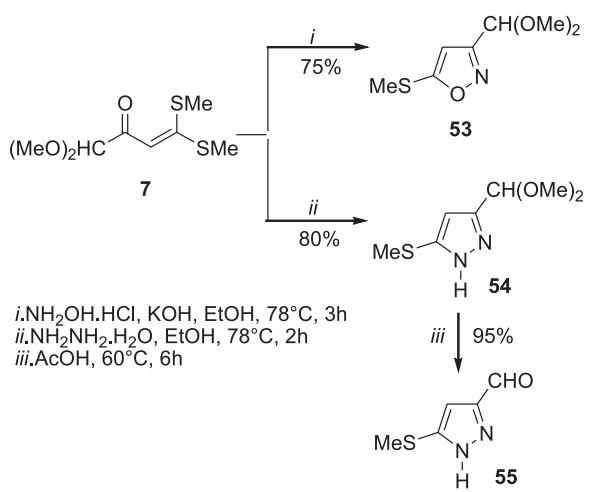

Esquema 21.

O tratamento do composto 7 com aminas levou à formação dos $\mathrm{N}, \mathrm{S}$-acetais (enaminonas) 56. O 5-aminoisoxazol 57 e o 5-aminopirazol $\mathbf{5 8}$ foram obtidos através da reação da enaminona $\mathbf{5 6}$ com hidroxilamina e hidrazina, respectivamente (Esquema 22). ${ }^{6}$ As reações levaram à formação exclusiva dos aminoazóis com a eliminação de tiometanol.

Esquema 22.
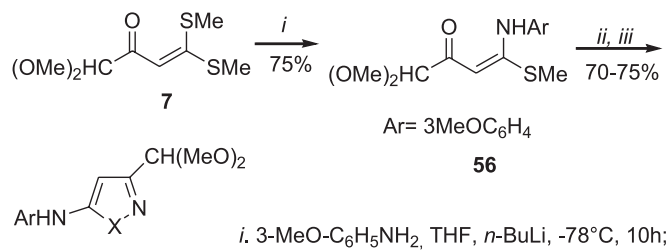

56

$\mathrm{X}=\mathrm{O} 57 \quad$ ii. $\mathrm{NH}_{2} \mathrm{NH}_{2} \cdot \mathrm{H}_{2} \mathrm{O}, \mathrm{EtOH}, 2 \mathrm{~h}$

NH 58 iii. $\mathrm{NH}_{2} \mathrm{OH} . \mathrm{HCl}, \mathrm{KOH}, \mathrm{EtOH}, 3 \mathrm{~h}$

Mellor e colaboradores, ${ }^{32}$ em 1997, sintetizaram 3-tiopirazóis 60 contendo o grupo mercapto a partir de reações do $\alpha$-oxoceteno ditioacetal 59 com hidrazina, metil-hidrazina e fenil-hidrazina (Esquema 23).

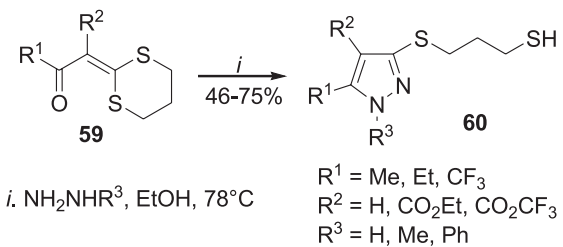

Esquema 23.

\section{Síntese de ditioltionas}

Curphey e colaboradores, ${ }^{33}$ em 2000, sintetizaram ditioltionas 62 através dos diânion do ácido 3-oxoditióico 61. Os diânions foram convertidos com bons rendimentos em 1,2-ditiol-3-tionas 62 pela ação do hexametildissililtiano (HMDT) e oxidação com hexacloroetano (Esquema 24).

Os autores ${ }^{33}$ acreditam que a transformação de diânions em ditioltionas procede conforme a sequência de passos apresentada na Figura 6.

\section{Síntese de oxazóis e imidazóis}

Abdelhaim e colaboradores ${ }^{7}$ descreveram a obtenção dos heterociclos benzoxazol $(X=\mathrm{O}) 63$ e benzoimidazol $(X=\mathrm{NH}) 64$ através 


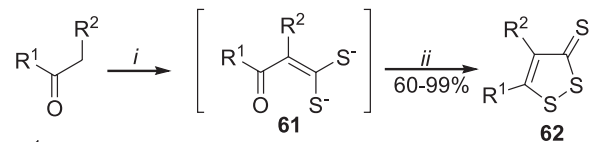

$\mathrm{R}^{1}=\mathrm{Ph}, 4-\mathrm{MeOPh}, \mathrm{t}-\mathrm{Bu},-\left(\mathrm{CH}_{2}\right)_{3^{-}},\left(\mathrm{CH}_{2}\right)_{4^{-}}, \mathrm{Me}$ $\mathrm{R}^{2}=\mathrm{H}, \mathrm{Me}, \mathrm{SMe},-\left(\mathrm{CH}_{2}\right)_{3^{-}},\left(\mathrm{CH}_{2}\right)_{4}$

i. a) $\mathrm{NaH} / \mathrm{THF}$. b) $\mathrm{CS}_{2} / \mathrm{THF} / \mathrm{DMPU}$ ii.a) $\mathrm{HMDT}$. b) $\mathrm{C}_{2} \mathrm{Cl}_{6}$

Esquema 24.

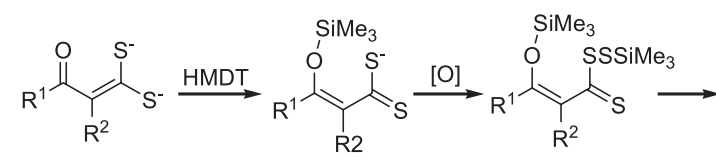

61<smiles>[R]C(=O)/C([R1])=C(/SC(C)C)S(C)(C)C</smiles>

62

Figura 6. Provável mecanismo de formação de 1,2-ditiol-3-tionas

da reação do $\alpha$-oxoceteno ditiometilacetal 9 com $o$-aminofenol e $o$-fenilenodiamina, respectivamente (Esquema 25). A reação procede pelo ataque inicial do nitrogênio do dinucleófilo ao carbono $\beta$ à carbonila, seguida da eliminação de uma molécula de tiometanol. Novo ataque do oxigênio ou nitrogênio do dinucleófilo ao carbono $\beta$ seguido de uma nova eliminação de um grupo tiometanol, produz os heterociclos 63 e 64.

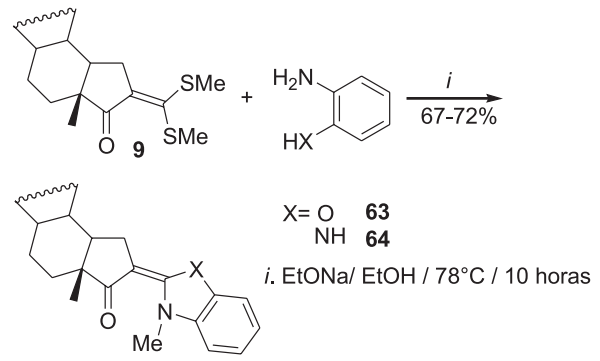

Esquema 25.

Síntese de anéis de seis membros

Sintese de piridinas

Potts e colaboradores ${ }^{34}$ estudaram a reação de $\alpha$-oxoceteno ditioacetais 65 com os enolatos de cetonas e posterior tratamento do intermediário com acetato de amônia e ácido acético para formar as 4-tioalquilpiridinas 66 (Esquema 26). Piridinas também foram obtidas através da reação dos $\alpha$-oxoceteno ditioacetais com o enol da acetonitrila (gerado in situ com $n$-butil-litio). ${ }^{35}$ Em condições reacionais semelhantes, piridinas 2,6-dissubstituídas ${ }^{36}$ e oligopiridinas também foram sintetizadas. ${ }^{37}$

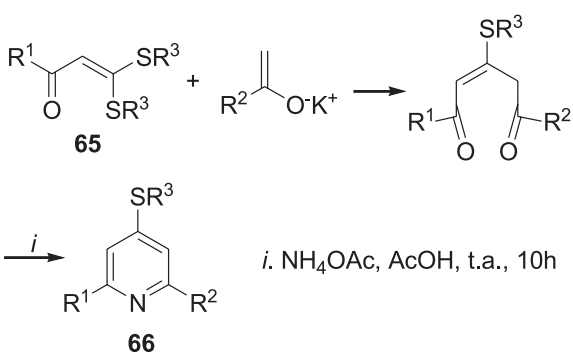

Esquema 26.
Thomas e Asokan ${ }^{38}$ desenvolveram em 2002, a partir de $\alpha$-oxoceteno ditioacetais, a síntese de 4-aril-2-tiometilpiridinas $\mathbf{7 0}$ tendo como precursor as 4,4-ditiometil-1-aril-3-propen-1-onas 67 (Esquema 27). O intermediário $\alpha$-hidroxiceteno ditiometilacetal 68 é sintetizado a partir da reação de adição à carbonila do composto 67 com o reagente de Grignard (preparado in situ com iodeto de metila e magnésio). Posteriormente, ocorre a desidratação do intermediário 68 induzido pela presença do reagente de Vilsmeier levando à formação in situ do 1,1-ditiometil-3-aril1,3-butadieno 69 que reage com o sal de iminoclorometileno na presença de acetato de amônio para formar as 2-tiometilpiridinas substituídas $\mathbf{7 0}$.
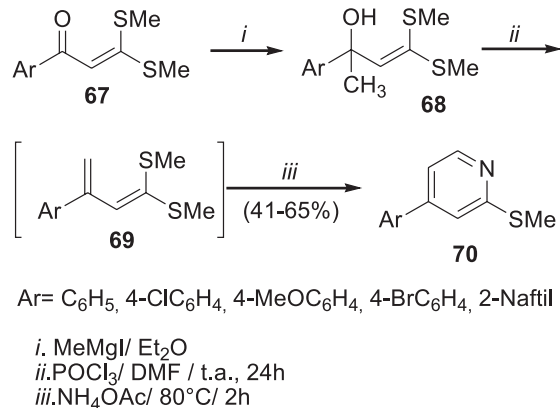

Esquema 27.

\section{Síntese de piranos}

Liu e colaboradores ${ }^{39}$ sintetizaram di-hidropiranos pela reação de $\alpha$-oxoceteno ditioacetais com $\mathrm{POCl}_{3}$-DMF, passando pelo intermediário 72 e posterior ciclização intramolecular à temperatura ambiente por $48 \mathrm{~h}$, formando os di-hidropiranos $\mathbf{7 3}$, com rendimento de $61-70 \%$ (Esquema 28).

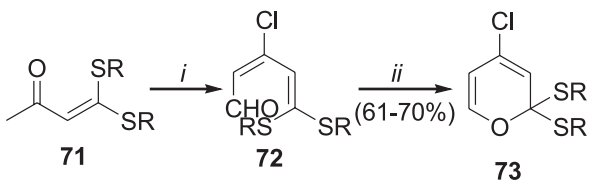

i. $\mathrm{POCl}$-DMF/ t.a./ $12 \mathrm{~h}$

ii. $\mathrm{Et}_{2} \mathrm{O} /$ t.a./ $48 \mathrm{~h}$

\section{Esquema 28.}

\section{$\underline{\text { Sintese de pirimidinas }}$}

Potts e colaboradore ${ }^{40}$ obtiveram pirimidinas $\mathbf{7 5}$ através da reação do $\alpha$-oxoceteno ditioacetal $\mathbf{7 4}$ com carboxiamidina em solução de benzeno e DMF na presença de hidreto de sódio, com rendimentos de moderados a bons (Esquema 29). A carboxiamidina geralmente é usada em forma de sal e a amidina livre, necessária para a reação, foi obtida através da adição de hidreto de sódio.

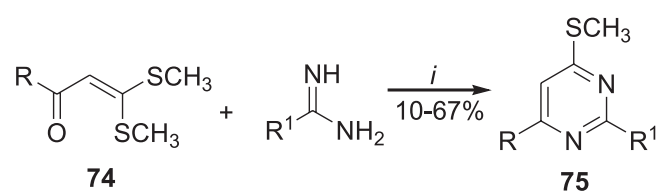

i. Benzeno-DMF/ NaH/ Refluxo 2h/ t.a. $16 \mathrm{~h}$

\section{Esquema 29.}

O trabalho de Mahata e colaboradores ${ }^{6}$ apresenta também a síntese da 2-aminopirimidina $\mathbf{7 5}$ em duas etapas. Primeiramente o precursor 7 reage com a guanidina ou a tioureia para formar a 2-aminopirimidina 76 ou a 2-mercaptopirimidina 77, respectivamente. A reação da 2-amino-4-alcoxi-6-(dimetoximetil)pirimidina $\mathbf{7 6}$ com solução de ácido clorídrico regenera o grupo aldeído formando a [(2-amino4-etoxi)pirimidin-6-il]carbaldeído 78 (Esquema 30). 
<smiles>[R]Oc1cc(C(OC)OC)nc([Y])n1</smiles>

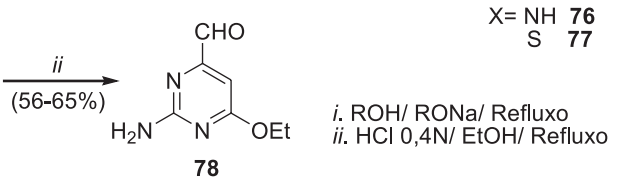

Esquema 30.

Mellor e colaboradores,$^{32}$ em 1997, sintetizaram 4-tiopirimidinas 80 contendo o grupo mercapto a partir de reações do $\alpha$-oxoceteno ditioacetal 79 com hidrazinas, guanidinas e tioureia (Esquema 31).

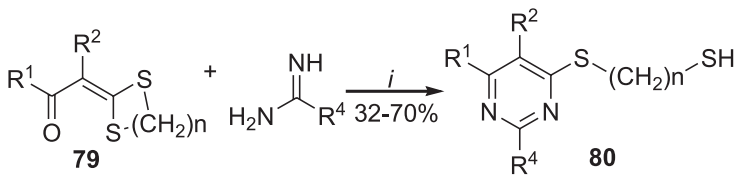

i. $\mathrm{K}_{2} \mathrm{CO}_{3}$, xileno, $140^{\circ} \mathrm{C}, 4 \mathrm{~h}$

$\mathrm{n}=2,3$

$\mathrm{R}^{1}=\mathrm{Me}, \mathrm{Et}, \mathrm{CF}_{3}$

$\mathrm{R}^{2}=\mathrm{H}, \mathrm{CO}_{2} \mathrm{Et}, \mathrm{CO}_{2} \mathrm{CF}_{3}$

$\mathrm{R}^{4}=\mathrm{SH}, \mathrm{NHEt}, \mathrm{NMe}_{2}, \mathrm{NEt}_{2}$

\section{Esquema 31.}

Mathews e colaboradores ${ }^{41}$ promoveram a síntese de 2-amino-6aril-4-(tiometil)pirimidin-5-il carbaldeído 81 a partir do $\alpha$-oxoceteno ditioacetal 32 e guanidina (Esquema 32). A formação das pirimidinas $\mathbf{8 1}$ mostrou ser dependente do solvente utilizado. As reações tendo acetonitrila como solvente apresentaram rendimentos superiores (70$82 \%$ ) quando comparadas àquelas reações em que a dimetilformamida foi o solvente (40-54\%). Embora as reações com DMF apresentassem rendimentos baixos, os autores concluíram que a técnica é limpa e simples para obter as carbaldeídopirimidinas.<smiles>COc1nc(N)nc([Al]C=CC(=O)OCC(C)(C)C)c1C=O</smiles>
ii. $\mathrm{K}_{2} \mathrm{CO}_{3} / \mathrm{CH}_{3} \mathrm{CN} / 100^{\circ} \mathrm{C} / 2 \mathrm{Oh}(70-82 \%)$

\section{Esquema 32.}

Abdelhaim e colaboradores ${ }^{7}$ descreveram a obtenção dos heterociclos pirimidinona 82 e tiopirimidinona 83 através da reação do $\alpha$-oxoceteno ditiometilacetal 9 com ureia e tioureia, respectivamente (Esquema 33). Além do estudo de síntese, descreveram a atividade antimicrobiana dos compostos sintetizados.

\section{Sintese de quinolinas}

Os $\alpha$-oxoceteno- $N, S$-arilaminoacetais (enaminonas) $\mathbf{8 5}$ foram sintetizados pela reação de anilinas substituídas com os $\alpha$-oxoceteno$S, S$-ditiometilacetais $\mathbf{8 4 . 4 2}$ As enaminonas 85 reagiram com solução do reagente de Vielsmeier (dimetilformamida- $\mathrm{POCl}_{3}$ ou dimetilacetamida- $\mathrm{POCl}_{3}$ ) através de uma ciclização intramolecular com eliminação de água, obtendo-se as 2-tiometilquinolinas $\mathbf{8 6}$ com rendimento de 25-98\% (Esquema 34). Além disso, os $\alpha$-oxoceteno ditioacetais 84 reagiram com a $o$-fenilenodiamina, a $m$-fenilenodiamina e o 1,5-diaminonaftaleno para produzir aminoquinolinas.

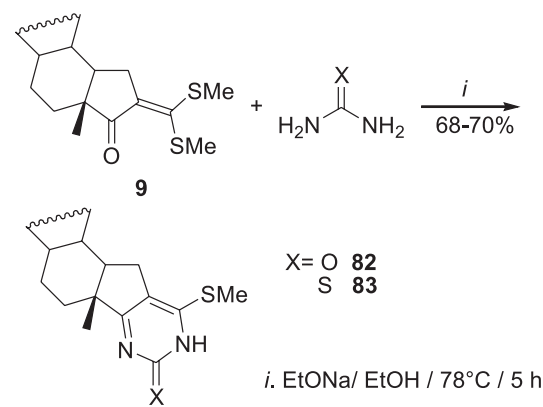

Esquema 33
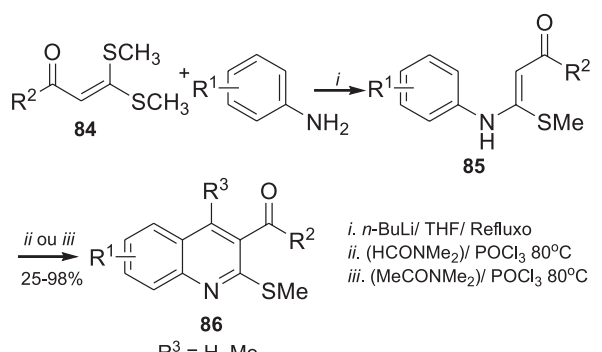

Esquema 34

Sintese de heterociclos condensados

Panda e colaboradores ${ }^{43}$ sintetizaram pirido[1,2-a]benzoimidazóis 90 e 91 envolvendo a ciclocondensação dos ânions do 2-metilbenzoimidazol e do 2-cianometilbenzoimidazol com uma variedade de $\alpha$-oxoceteno ditioacetais 87. O ânion derivado do 2-metilbenzoimidazol $\mathbf{8 8}$ promoveu adição regiosseletiva, atacando a carbonila do $\alpha$-oxoceteno ditioacetais 87. Já na reação do anion derivado do 2-cianometilbenzoimidazol 89 com os $\alpha$-oxoceteno ditioacetais 87 a adição ocorreu seletivamente no carbono $\beta$ à carbonila (Esquema 35). Piridoindóis também foram sintetizados através da reação do enolato do 1-metil-2-oxoindol com $\alpha$-oxoceteno ditioacetais com rendimentos moderados a bons. ${ }^{44}$

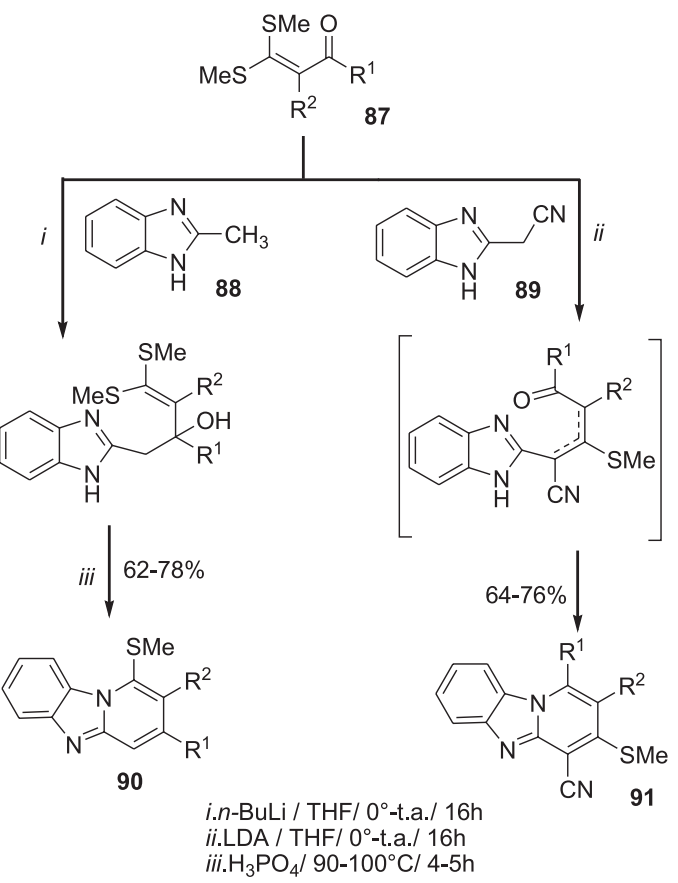

Esquema 35.

Suresh e colaboradores, ${ }^{45} \mathrm{em} 2000$, sintetizaram os benzotiofenos 93 e 94 através da reação entre 2-tiofenoacetonitrila e 3-tiofenoace- 
tonitrila, respectivamente (Esquema 36). A reação se dá pela perda do próton $\alpha$ ao grupo ciano, dos 3- ou 2-tiofenoacetonitrila, gerando um nucleófilo que ataca o carbono $\beta$ do $\alpha$-oxoceteno ditiometilacetais 92 que, por posterior ciclização intramolecular e eliminação de um grupo tiometanol, produz os benzotiofenos 93 e 94 .

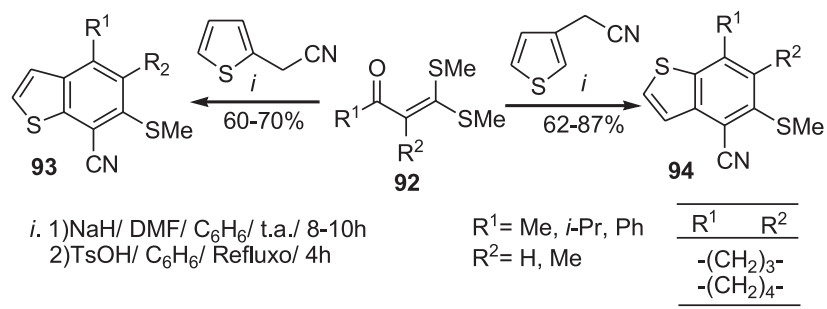

Esquema 36.

Peruncheralathan e colaboradore ${ }^{46}$ sintetizaram os tiometilindazóis 97 em duas etapas, por meio de uma reação envolvendo a adição do 1,3-difenil-5-cianometilpirazol aos $\alpha$-oxocetenos ditiometilacetais 95 (Esquema 37). Os indazóis 97 foram obtidos através da reação de ciclização intramolecular promovida pela reação do intermediário 96 com o ácido $p$-toluenossulfônico. O enolato derivado do 4-cianometil2-feniltiazol também foi utilizado como precursor para a síntese de tioalquilbenzotiazois apresentando rendimentos moderados. ${ }^{47}$
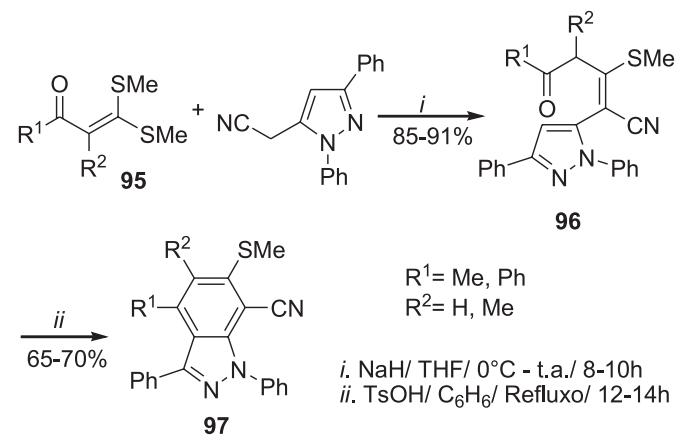

Esquema 37.

Kishore e colaboradores, ${ }^{48}$ em 1999 , promoveram a síntese de pirazolo[1,5- $a$ ]piridinas $\mathbf{1 0 0}$ através da reação do lítio pirazol-2carboxilato formado in situ com o $\alpha$-oxoceteno ditiometilacetal 98. O reagente organometálico adiciona-se à carbonila dos $\alpha$-oxoceteno ditioacetais formando o intermediário 99, que sofre ciclização intramolecular com eliminação de $\mathrm{CO}_{2}$ e MeSH, seguida de desidratação para formar o pirazolopiridina $\mathbf{1 0 0}$ em bons rendimentos (Esquema 38). Benzoisoxazóis foram sintetizados de maneira semelhante, tendo como precursor o 5-litiometil-3-metilisoxazol (gerado in situ). ${ }^{49}$
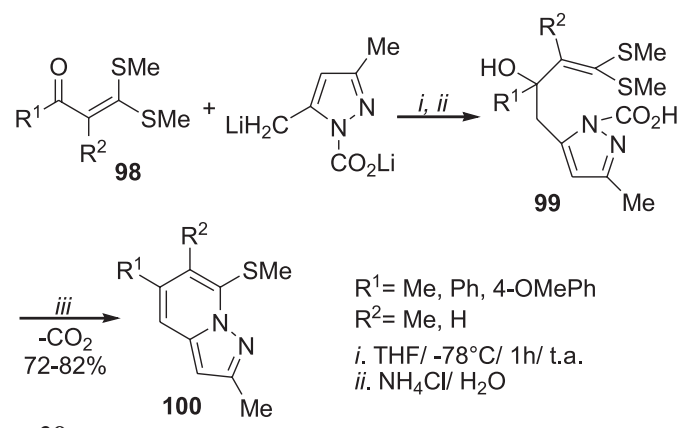

$\mathrm{R}^{1}=\mathrm{Me}, \mathrm{Ph}, 4-\mathrm{OMePh}$

$\mathrm{R}^{2}=\mathrm{Me}, \mathrm{H}$

i. $\mathrm{THF} /-78^{\circ} \mathrm{C} / 1 \mathrm{~h} / \mathrm{t} . \mathrm{a}$.

Esquema 38.

ii. $\mathrm{NH}_{4} \mathrm{Cl} / \mathrm{H}_{2} \mathrm{O}$

Balu e colaboradore ${ }^{50}$ realizaram a reação de anelação de naftalenos partindo dos $\alpha$-oxoceteno ditiometilacetais com a eliminação de ambos os grupos tioalquilas. O trabalho realizado por Mehta e colaboradores ${ }^{15}$ proporcionou a manutenção de um dos grupos tiometil no naftaleno. A reação de $\alpha$-oxoceteno ditiometilacetal 101 com os reagentes de Grignard cloreto de 4-metoxibenzilmagnésio $\left(\mathrm{R}^{2}=\mathrm{H}\right)$ e cloreto de 3,4-dimetoxibenzilmagnésio $\left(\mathrm{R}^{2}=\mathrm{OMe}\right)$ formou os $\alpha$-tiometilnaftalenos 103 (Esquema 39).<smiles>[R]c1cc(CC([R1])(O)/C=C(\C)S(C)(=O)=O)ccc1OC</smiles><smiles>[R]c1cc([As])c2cc(OC)c([R])cc2c1</smiles>

$\mathrm{R}^{1}=\mathrm{Me}, \mathrm{Ph}, 4-\mathrm{MePh}$ $\mathrm{R}^{2}=\mathrm{H}, \mathrm{OMe}$

i. $\mathrm{Et}_{2} \mathrm{O} / \mathrm{THF} / 0^{\circ} \mathrm{C}-$ t.a ii. $\mathrm{NH}_{4} \mathrm{Cl} / \mathrm{H}_{2} \mathrm{O}$ iii. $\mathrm{BF}_{3} . \mathrm{Et}_{2} \mathrm{O} / \mathrm{C}_{6} \mathrm{H}_{6} /$ Refluxo

Esquema 39.

\section{CONCLUSÃO}

Esta revisão mostrou a importância de $\alpha$-oxoceteno ditioacetais como intermediários em síntese orgânica, tanto por sua variedade de centros reativos como pela sua utilização como precursor na síntese de compostos heterocíclicos funcionalizados. As reações envolvem bons rendimentos e procedimentos experimentais relativamente simples, priorizando técnicas que permitem a introdução direta de átomos de enxofre na estrutura dos compostos. A presença de grupos tioalquil, tioaril ou do átomo de enxofre na estrutura desses heterociclos pode acrescentar uma variação no potencial biológico destes compostos.

\section{REFERÊNCIAS}

1. Konaklieva, M. I.; Plotkin, B. J.; Recent Pat. Antiinfect. Drug Discov. 2006, $1,177$.

2. Cunico, W.; Gomes, C. R. B.; Vellasco Jr., W. T.; Quim. Nova 2008, 31, 2111.

3. Peruncheralathan, S.; Khan, T. A.; Ila, H.; Junjappa, H.; J. Org. Chem. 2005, 70, 10030; Munday, R.; Munday, C. M.; Carcinogenesis 2004, 25, 1721.

4. Hojo, M.; Masuda, R.; J. Org. Chem. 1975, 40, 963.

5. Ali, S. M.; Tanimoto, S.; J. Chem. Soc., Chem. Commun. 1989, 684.

6. Mahata, P. K.; Syam Kumar, U. K.; Sriram, V.; Ila, H.; Junjappa, H.; Tetrahedron 2003, 59, 2631.

7. Abdelhalim, M. M.; El-Saidi, M. M. T.; Rabie, S. T.; Elmegeed, G. A.; Steroids 2007, 72, 459

8. Martins, M. A. P.; Pereira, C. M. P.; Zimmermann, N. E. Z.; Moura, S.; Sinhorin, A. P.; Cunico, W.; Zanatta, N.; Bonacorso, H. G.; Flores, A. C. F.; Synthesis 2003, 2353.

9. Ferraz, H. M. C.; Pereira, F. L. C.; Quim. Nova 2004, 27, 89; Ferraz, H. M. C.; Gonçalo, E. R. S.; Quim. Nova 2007, 30, 957.

10. Masson, S.; Thuillier, A.; Tetrahedron Lett. 1980, 21, 4085.

11. Gupta, A. K.; Ila, H.; Junjappa, H.; Tetrahedron 1989, 45, 1509.

12. Gammill, R. B.; Sobieray, D. M.; Gold, P. M.; J. Org. Chem. 1981, 46, 3555 .

13. Yadav, K. M.; Suresh, J. R.; Patro, B.; Ila, H.; Junjappa, H.; Tetrahedron 1996, 52, 4679.

14. Dieter, R. K.; Silks, L. A.; Fishpaugh, J. A.; Kastner, M. E.; J. Am. Chem. Soc. 1985, 107, 4679.

15. Mehta, B. K.; Nandi, S.; Ila, H.; Junjappa, H.; Tetrahedron 1999, 55 , 12843 . 
16. Yadav, K. M.; Mohanta, P. K.; Ila, H.; Junjappa, H.; Tetrahedron 1996 $52,14049$.

17. Pandey, S.; Suryawanshi, S. N.; Gupta, S.; Srivastava, V. M. L.; Eur. J. Med. Chem. 2005, 40, 751.

18. Yamamoto, M.; Furusawa, A.; Iwasa, S.; Kohmoto, S.; Yamada, K.; Bull Chem. Soc. Jpn. 1992, 65, 1550.

19. Anabha, E. R.; Asokan, C. V.; Synthesis 2006, 151.

20. Ortega-Jiménez, F.; López-Cortés, J. G.; Ortega-Alfaro, M. C.; Toscano, A.; Penieres, G.; Quijada, R.; Alvarez, C.; J. Organomet. Chem. 2005, $690,454$.

21. Nair, S. K.; Jose, A. M.; Asokan, C. V.; Synthesis 2005, 1261.

22. Wang, J.-S.; Shen, X.; He, X.; Zhu, Y.-R.; Zhang, B.-C.; Wang, J.-B.; Qian, G.-S.; Kuang, S.-Y.; Zarba, A.; Egner, P. A.; Jacobson, L. P.; Munoz, A.; Helzlsouer, K. J.; Groopman, J. D.; Kensler, T. W.; Natl. Cancer Inst. 1999, 91, 347.

23. Chevreuil, F.; Landreau, A.; Seraphin, D.; Larcher, G. ; Bouchara, J-P. ; Richomme, P.; J. Enzym. Inhib. Med. Ch. 2006, 21, 293.

24. Ondetti, M. A.; Rubin, B.; Cushman, D. W.; Science 1977, 196, 441.

25. Martins, M. A. P.; Pereira, C. M. P.; Zimmermann, N. E. Z.; Cunico, W.; Moura, S.; Beck, P.; Zanatta, N.; Bonacorso, H. G.; J. Fluorine Chem. 2003, 123, 261.

26. Martins, M. A. P.; Cunico, W.; Brondani, S.; Peres, R. L.; Zimmermann, N.; Rosa, F. A.; Fiss, G. F.; Zanatta, N.; Bonacorso, H. G.; Synthesis 2006, 1485.

27. Balu, M. P.; Ila, H.; Junjappa, H.; Tetrahedron 1990, 46, 6771.

28. Okazaki, R.; Negishi, Y.; Inamoto, N.; J. Org. Chem. 1984, 49, 3819.

29. Datta, A.; Pooranchand, D.; Ila, H.; Junjappa, H.; Tetrahedron 1989, 45, 7631.

30. Bhat, L.; Thomas, A.; Ila, H.; Junjappa, H.; Tetrahedron 1992, 48, 10377.

31. Banerjee, A. K.; Bandyopadhyay, S.; Gayen, A. K.; Sengupta, T.; Das, A. K.; Chatterjee, G. K.; Chaudhuri, S. K; Arzneimittel-Forschung (Drug Res.) 1994, 44, 863.
32. Mellor, J. M.; Schofield, S. R.; Korn, S. R.; Tetrahedron 1997, 53, 17163.

33. Curphey, T. J.; Libby, A. H.; Tetrahedron Lett. 2000, 41, 6977.

34. Potts, K. T.; Winslow, P. A; J. Org. Chem. 1985, 50, 5405.

35. Gupta, A. K.; Ila, H.; Junjappa, H.; Tetrahedron 1990, 46, 3703.

36. Potts, K. T.; Cipullo, M. J.; Ralli, P.; Theodoridis, G.; J. Org. Chem. 1982, 47, 3027.

37. Potts, K. T.; Raiford, K. A. G.; Keshavarz-K., M.; J. Am. Chem. Soc. 1993, 115, 2793.

38. Thomas, A. D.; Asokan, C. V.; Tetrahedron Lett. 2002, 43, 2273.

39. Liu, Y.; Dong, D.; Liu, Q.; Qi, Y.; Wang, Z.; Org. Biomol. Chem. 2004, $2,28$.

40. Potts, K. T.; Cipullo, M. J.; Ralli, P.; Theodoridis, G.; J. Org. Chem. 1983, 48, 4841.

41. Mathews, A.; Asokan, C. V.; Tetrahedron 2007, 63, 7845.

42. Mahata, P. K.; Venkatesh, C.; Syam Kumar, U. K.; Ila, H.; Junjappa, H.; J. Org. Chem. 2003, 68, 3966.

43. Panda, K.; Suresh, J. R.; Ila, H.; Junjappa, H.; J. Org. Chem. 2003, 68, 3498.

44. Barun, O.; Patra, P. K.; Ila, H.; Junjappa, H.; Tetrahedron Lett. 1999, 40, 3797.

45. Suresh, J. R.; Barun, O.; Ila, H.; Junjappa, H.; Tetrahedron 2000, 56, 8153.

46. Peruncheralathan, S.; Khan, T. A.; Ila, H.; Junjappa, H.; Tetrahedron 2004, 60, 3457.

47. Hegde, V. S.; Kolavi, G. D.; Khazi, I. A. M.; Synthetic Commun. 2006, 36, 2983.

48. Kishore, K.; Reddy, K. R.; Suresh, J. R.; Ila, H.; Junjappa, H.; Tetrahedron 1999, 55, 7645.

49. Pooranchand, D.; Satyanarayana, J.; Ila, H.; Junjappa, H.; Synthesis 1993, 241

50. Balu, M. P.; Singh, G.; Ila, H.; Junjappa, H.; Tetrahedron Lett. 1986, 27 , 117. 